\title{
Stabilization of discrete port-Hamiltonian dynamics via interconnection and damping assignment
}

\author{
Alessio Moreschini ${ }^{1,2}$, Mattia Mattioni ${ }^{1}$, Salvatore Monaco $^{1}$ and Dorothée Normand-Cyrot ${ }^{2}$
}

\begin{abstract}
The paper deals with interconnection and damping assignment for discrete-time port-Hamiltonian systems. Based on a novel state representation, suitably shaped to address energybased control design, the nonlinear discrete-time controller is characterized and the solution is explicitly computed in the linear case. The design worked out on the exact sampled-data model of a mechanical system confirms the effectiveness of the controller.
\end{abstract}

Index Terms-Energy systems, Lyapunov methods, Sampleddata control.

\section{INTRODUCTION}

$\mathbf{P}$ ORT-HAMILTONIAN dynamics introduced in [1] encompass a broad class of physical systems including mechanical, electrical, hydraulic, and many others [2]. They are issued from network-inspired modeling and concern dynamics endowed with a Hamiltonian function along with power-conserving interconnection and energy dissipating components. As well known, port-Hamiltonian systems provide a natural setup for the development of passivity-based control (PBC) as well as strategies based on energy management, as Energy Shaping (ES) and Interconnection and Damping Assignment (IDA-PBC), widely discussed in [3]-[5] and references therein.

Port-Hamiltonian representations and energy-based control strategies have essentially been discussed in continuous time what is often not sufficient in practice for real-time applications, as haptics or telemanipulation among others. In fact, physical processes require a Hamiltonian reformulation in a discrete-time framework so allowing for the design of ad hoc digital control laws.

In spite of a growing interest of the community for modeling Hamiltonian structures in discrete time in various mathematical set-ups [6]-[14], a well identified state-space representation endorsing the required interconnection and energy balance properties is still not available, in particular for developing control-oriented designs. However, a shared basic tool is the discrete gradient function [15] which properly expresses the variation of a function (the Hamiltonian) between two points.

Supported by Université Franco-Italienne/Università Italo-Francese (Vinci Grant 2019) and by Sapienza Università di Roma (Progetti di Ateneo 2018Piccoli progetti RP11816436325B63).

${ }^{1}$ Dipartimento di Ingegneria Informatica, Automatica e Gestionale A. Ruberti (Sapienza University of Rome); Via Ariosto 25, 00185 Rome, Italy \{alessio.moreschini, mattia.mattioni, salvatore.monaco\}euniromal.it.

${ }^{2}$ Laboratoire des Signaux et Systèmes (L2S-CNRS-UMR 8506); 3, Rue Joliot Curie, 91192, Gif-sur-Yvette, France \{alessio.moreschini, dorothee.normand-cyrot \}acentralesupelec.fr
Accordingly, the proposed discrete-time dynamics [7]-[14] are suitably shaped to model the power change in terms of dissipated and supplied energy although many difficulties inherent to the discrete-time context remain: the state-space dynamics is unavoidably implicitly defined, the notion of passivity invoked to define a power conjugate output is unclear and the invariance of the form under feedback is a challenging problem. Partial solutions to these problems are only described in the literature even for discrete-time dynamics issued from sampling that by construction somehow match the energetic behavior of the continuous-time form [6], [16], [17]. In that case, the solutions developed are approximate and strongly sensitive to the sampling period.

To handle these difficulties, a novel representation of port Hamiltonian dynamics in a purely discrete-time context has been proposed by the authors in [18]. In particular, such form has been shown to be suitably shaped to define a conjugate passivating output relying on the notion of discrete-time average passivity introduced in [19]. An average passivitybased feedback has also been shown in [18] to get asymptotic stability by increasing the damping.

It must be noted that the adequacy of these forms is reinforced by a sampled-data oriented study which shows that such representations are recovered under exact sampling thanks to suitably constructed interconnection and damping matrices depending on the sampling period. This is discussed in [20] when referring to a quadratic Hamiltonian function and constant interconnection and damping matrices.

In the present paper, we further exploit the discrete-time structure proposed in [18] to discuss energy management strategies. More precisely, we set the problem of asymptotic stabilization towards a target equilibrium that corresponds to the energy minimum of a new Hamiltonian function. Following the lines of the continuous-time set-up [3], [4], the question is formulated in terms of feedback passivation with respect to a new Hamiltonian function with possibly new interconnection and damping components. Since in discrete time the main difficulty stands in the preservation of the Hamiltonian structure under feedback, the notion of admissible equilibria is introduced. Then, the problem solvability is lead to the solution of a nonlinear algebraic equation. The proposed controller is further specified to the linear-time invariant (LTI) case where a necessary and sufficient condition is given together with an explicit feedback solution. Finally, the IDA-PBC controller is computed on the exact sampleddata equivalent model of a mechanical case study allowing a comparison with the literature [9]-[11]. 
The paper is organized as follows. The problem formulation is given in Section II. Section III discusses the discrete IDAPBC approach for nonlinear discrete-time port-Hamiltonian dynamics. In Section IV the approach is developed for LTI systems. In Section V the proposed control scheme is applied to the sampled-data equivalent dynamics of a mechanical example and compared to the present literature. Concluding remarks are in Section VI.

Notations: Functions and vector fields are assumed smooth and complete over the respective definition spaces. $\mathbb{R}$ and $\mathbb{N}$ denote the set of real and natural numbers including 0 . For any vector $z \in \mathbb{R}^{n},\|z\|$ and $z^{\top}$ define respectively the norm and transpose of $z$. The superscript $\dagger$ denotes the pseudoinverse, $z^{\dagger}=\left(z^{\top} z\right)^{-1} z^{\top}$. Given a square matrix $R$ and $z \in \mathbb{R}^{n}$, the weighted square norm is defined as $\|z\|_{R}^{2}:=z^{\top} R z . I_{d}$ denotes the identity matrix. Given a twice continuously differentiable function $S(\cdot): \mathbb{R}^{n} \rightarrow \mathbb{R}, \nabla S$ represents its gradient vector and $\nabla^{2} S$ its Hessian matrix.

\section{ReCAlls AND PROBlem FORMULATION}

\section{A. Discrete port-Hamiltonian system}

The notion of discrete gradient function is first recalled from [15], [21].

Definition 2.1 (Discrete gradient): Given $S(\cdot): \mathbb{R}^{n} \rightarrow \mathbb{R}$, a smooth real-valued function, its discrete gradient is a vectorvalued function of two variables $\bar{\nabla} S(v, w): \mathbb{R}^{n} \times \mathbb{R}^{n} \rightarrow$ $\mathbb{R}^{n}$ (denoted $\left.\bar{\nabla} S\right|_{v} ^{w}:=\bar{\nabla} S(v, w)=\operatorname{col}\left(\left.\left.\bar{\nabla}_{1} S\right|_{v} ^{w} \cdots \bar{\nabla}_{n} S\right|_{v} ^{w}\right)$ ) satisfying for all $v, w \in \mathbb{R}^{n}$ the variational equality

$$
\left.(w-v)^{\top} \bar{\nabla} S\right|_{v} ^{w}=S(w)-S(v),
$$

with $\left.\bar{\nabla} S\right|_{v} ^{v}=\nabla S(v)$ set for continuity argument.

According to Definition 2.1, for $v=\operatorname{col}\left(v_{1}, \ldots, v_{n}\right)$ and $w=\operatorname{col}\left(w_{1}, \ldots, w_{n}\right)$, the discrete gradient function can be computed component-wise through the integral form

$$
\left.\bar{\nabla}_{i} S\right|_{v} ^{w}=\frac{1}{w_{i}-v_{i}} \int_{v_{i}}^{w_{i}} \frac{\partial S\left(w_{1}, \ldots, w_{i-1}, \xi, v_{i+1}, \ldots, v_{n}\right)}{\partial \xi} \mathrm{d} \xi
$$

The discrete port-Hamiltonian form we deal with has been recently proposed in [18].

Definition 2.2: A discrete single-input/single-output portHamiltonian system with smooth Hamiltonian function $H(\cdot)$ : $\mathbb{R}^{n} \rightarrow \mathbb{R}$ is described as

$$
\begin{aligned}
x^{+}(u) & =x+\left.(J(x)-R(x)) \bar{\nabla} H\right|_{x} ^{x^{+}}+g(x, u) u \\
y(x, u) & =\left.g^{\top}(x, u) \bar{\nabla} H\right|_{x^{+}} ^{x^{+}(u)}
\end{aligned}
$$

with $u \in \mathbb{R}, x \in \mathbb{R}^{n}$, controlled dynamics $x^{+}(u)$, and drift dynamics $x^{+}:=x^{+}(0)$ implicitly described by

$$
x^{+}=x+\left.(J(x)-R(x)) \bar{\nabla} H\right|_{x} ^{x^{+}}
$$

where $J(x)=-J^{\top}(x)$ and $R(x)=R^{\top}(x) \succeq 0$ are the interconnection and damping matrices.

In the form (1), time-dependencies are dropped out for the ease of notation. For all $k \geq 0$, we denote $x:=x_{k}, u:=u_{k}$, so that (1a) defines the one-step ahead state evolution that is $x^{+}(u)=x_{k}^{+}\left(u_{k}\right):=x_{k+1}$.
It must be pointed out that equation (1a) does not provide an explicit representation for the drift, $x^{+}$, and it is generically nonlinear with respect to the control variable $u$. Moreover, passivity holds with respect to the so defined conjugate output map (1b). To analyze this aspect, the one step variation of the Hamiltonian function is computed as

$$
\begin{aligned}
& \Delta H(x):=H\left(x^{+}(u)\right)-H(x) \\
& =\left.\left.\bar{\nabla}^{\top} H\right|_{x} ^{x^{+}}(J(x)-R(x)) \bar{\nabla} H\right|_{x} ^{x^{+}}+\left.\bar{\nabla}^{\top} H\right|_{x^{+}} ^{x^{+}(u)} g(x, u) u
\end{aligned}
$$

with in particular, $\left\|\left.\bar{\nabla} H\right|_{x} ^{x^{+}}\right\|_{J}^{2} \equiv 0$. The equality above is at the basis of the properties below.

1) Any critical point $x_{e}$ of the Hamiltonian $H(x)$, namely $\left.\bar{\nabla} H\right|_{x_{e}} ^{x_{e}}=\nabla H\left(x_{\star}\right)=0$ is an equilibrium point of (1a). $x_{e}$ is said to be non-degenerate if $\nabla H^{2}\left(x_{e}\right) \neq 0$.

2) If $H\left(x_{e}\right)<H(x)$ for all $x$ in the neighborhood of $x_{e}$, then the equilibrium point $x_{e}$ is stable with Lyapunov function $H(x)$; moreover, it is asymptotically stable if the largest invariant set contained in $\{x \in$ $\mathbb{R}^{n}\left|\left\|\left.\bar{\nabla} H\right|_{x} ^{x^{+}}\right\|_{R}^{2}=0\right\}$ is $\left\{x_{e}\right\}$.

3) Qualifying $H(x)$ as a storage function, the dynamics (1a) is passive with respect to the conjugate output (1b) with dissipation inequality

$$
\begin{aligned}
\Delta H(x)= & -\left\|\left.\bar{\nabla} H\right|_{x} ^{x^{+}}\right\|_{R}^{2}+\left.\bar{\nabla}^{\top} H\right|_{x^{+}} ^{x^{+}(u)} g(x, u) u \\
& \leq\left. u g^{\top}(x, u) \bar{\nabla} H\right|_{x^{+}} ^{x^{+}(u)}
\end{aligned}
$$

and, for any $k \geq 0$, discrete energy balance equality

$$
\begin{aligned}
\underbrace{H\left(x_{k}\right)-H\left(x_{0}\right)}_{\text {stored energy }} & =\underbrace{\left.\sum_{i=0}^{k-1} \bar{\nabla}^{\top} H\right|_{x_{i}^{+}} ^{x_{i}^{+}\left(u_{i}\right)} g\left(x_{i}, u_{i}\right) u_{i}}_{\text {supplied energy }} \\
& \underbrace{-\left.\left.\sum_{i=0}^{k-1} \bar{\nabla} \bar{\nabla}^{\top} H\right|_{x_{i}} ^{x_{i}^{+}} R\left(x_{i}\right) \bar{\nabla} H\right|_{x_{i}} ^{x_{i}^{+}}}_{\text {dissipated energy }} .
\end{aligned}
$$

Differently from those in the literature, the proposed portHamiltonian system (1) allows to separate into the energy balance equation (EBE) the total dissipated energy from the supplied one.

\section{B. Problem statement}

The control objective we address stands in the assignment of a given equilibrium while preserving the port-Hamiltonian structure. More precisely, the problem consists in stabilizing the port-Hamiltonian system (1a) at a desired equilibrium point, say $x_{\star}$, by possibly assigning through feedback a new Hamiltonian function $H_{d}(x)$ with a minimum in $x_{\star}$. As not all $x_{\star} \in \mathbb{R}^{n}$ can be assigned to (1a), the class of admissible equilibria must be characterized. For, we can give the following definition.

Definition 2.3: $x_{\star} \in \mathbb{R}^{n}$ is an admissible equilibrium for (1a) if it is contained in the set

$$
\begin{array}{r}
\mathcal{E}:=\left\{x \in \mathbb{R}^{n} \mid g^{\perp}(x, u)(J(x)-R(x)) \nabla H(x)=0,\right. \\
\text { for some } u \in \mathbb{R}\},
\end{array}
$$


with $g^{\perp}(x, u)$ denoting the left-hand side annihilator of $g(x, u)$ (i.e., $g^{\perp}(x, u) g(x, u)=0$, for all $\left.(x, u) \in \mathbb{R}^{n} \times \mathbb{R}\right)$. Any $u$ corresponding to $x_{\star}$ is denoted by $u_{\star}$.

On this basis, the following discrete-time IDA-PBC problem can be formulated.

DT-IDA-PBC problem: Given (1a) and $x_{\star} \in \mathcal{E}$, find $\bar{u}(x)$ : $\mathbb{R}^{n} \rightarrow \mathbb{R}$ verifying $u_{\star}=\bar{u}\left(x_{\star}\right)$ such that for $u=\bar{u}(x)+v$ the system takes the port-Hamiltonian form

$$
x^{+}(\bar{u}(x)+v)=x+\left.\left(J_{d}(x)-R_{d}(x)\right) \bar{\nabla} H_{d}\right|_{x} ^{x^{+}(\bar{u}(x))}+g_{d}(x, v) v
$$

with new Hamiltonian $H_{d}: \mathbb{R}^{n} \rightarrow \mathbb{R}_{\geq 0}$ possessing a local minimum at $x_{\star}, J_{d}(x)=-J_{d}^{\top}(x), R_{d}(x)=R_{d}^{\top}(x) \succeq 0$ and $g_{d}(x, v)$ satisfying

$v g_{d}(x, v)=\bar{u}(x)(g(x, \bar{u}(x)+v)-g(x, \bar{u}(x)))+v g(x, \bar{u}(x)+v)$.

Accordingly, the closed-loop (2) verifies the new energy balance equality

$$
H_{d}\left(x_{k}\right)-H_{d}\left(x_{0}\right)=\sum_{i=0}^{k-1} v_{i} y_{d_{i}}-\sum_{i=0}^{k-1} w_{d_{i}}, \forall k \in \mathbb{N},
$$

with dissipation $w_{d} \geq 0$ and new passive output

$$
y_{d}(x, v)=\left.g_{d}^{\top}(x, v) \bar{\nabla} H_{d}\right|_{x^{+}(\bar{u}(x))} ^{x^{+}(\bar{u}(x)+v)} \triangleleft \triangleleft
$$

It is worth to note that the problem of recovering the Hamiltonian structure (2) under feedback is challenging because of the dependency in $x^{+}(\bar{u}(x))$ of the discrete-gradient $\left.\bar{\nabla} H_{d}\right|_{x} ^{x^{+}(\bar{u}(x))}$. For the ease of notation, in the following $\bar{u}(x)$ is denoted by $\bar{u}$.

\section{IDA-PBC IN DISCRETE TIME}

The solvability of the DT-IDA-PBC problem for dynamics of the form (1a) is discussed in this section. A first characterization of the solution is given below when the interconnection and damping matrices are not modified (i.e., $J_{d}(x)=J(x)$ and $R_{d}(x)=R(x)$ ).

Proposition 3.1: Given (1a) and $x_{\star} \in \mathcal{E}$, the DT-IDA-PBC problem is solvable for $J_{d}(x)=J(x)$ and $R_{d}(x)=R(x)$, if there exist $H_{d}(\cdot): \mathbb{R}^{n} \rightarrow \mathbb{R}_{\geq 0}$ verifying

(i) $\nabla H\left(x_{\star}\right)=-\nabla H_{a}\left(x_{\star}\right)$

(ii) $H\left(x_{\star}\right)-H(x)<H_{a}(x)-H_{a}\left(x_{\star}\right) \quad \forall x \in \mathcal{B} /\left\{x_{\star}\right\}$, with $H_{a}(x)=H_{d}(x)-H(x)$ and $\mathcal{B}$ an open neighborhood of $x_{\star}$ and a feedback $\bar{u}(x)$ satisfying $u_{\star}=\bar{u}\left(x_{\star}\right)$ solution to the matching equation

$$
\begin{array}{r}
(J(x)-R(x))\left(\left.\bar{\nabla} H_{a}\right|_{x} ^{x^{+}(\bar{u})}+\left.\bar{\nabla} H\right|_{x} ^{x^{+}(\bar{u})}\right. \\
\left.-\left.\bar{\nabla} H\right|_{x} ^{x^{+}}\right)=g(x, \bar{u}) \bar{u} .
\end{array}
$$

Moreover, $x_{\star} \in \mathcal{E}$ is a stable equilibrium for the closed-loop port-Hamiltonian dynamics

$$
x^{+}(\bar{u})=x+\left.(J(x)-R(x)) \bar{\nabla} H_{d}\right|_{x} ^{x^{+}(\bar{u})}
$$

with Lyapunov function $H_{d}(x) . x_{\star}$ is asymptotically stable if the largest invariant set contained in $\{x \in$ $\mathbb{R}^{n}\left|\left\|\left.\bar{\nabla} H_{d}\right|_{x} ^{x^{+}(\bar{u})}\right\|_{R}^{2}=0\right\}$ is $\left\{x_{\star}\right\}$.
Proof. According to $(i), H_{d}(x)$ has a critical point at $x_{\star}$ while (ii) provides that $x_{\star}$ is a minimum. The feedback $\bar{u}(x)$ solution to (5) achieves the target dynamics (6) by construction. Moreover, by specifying (5) at $x=x_{\star}$ and $u_{\star}=\bar{u}\left(x_{\star}\right)$ one gets from $(i)$,

$$
-\left(J\left(x_{\star}\right)-R\left(x_{\star}\right)\right) \nabla H\left(x_{\star}\right)=g\left(x_{\star}, \bar{u}\left(x_{\star}\right)\right) \bar{u}\left(x_{\star}\right)
$$

which is satisfied only if $x_{\star} \in \mathcal{E}$. Finally, by qualifying $H_{d}(x)$ as a Lyapunov function for (6), (asymptotic) stability of $x_{\star}$ follow from (i), (ii) in Proposition 3.1 and the property 2) of the discrete Hamiltonian form as in Section II.A.

Allowing to modify the interconnection and damping matrices enlarges the set of possible solutions that assign the desired equilibrium so getting the following result.

Proposition 3.2: Consider (1a) and $x_{\star} \in \mathcal{E}$, the DT-IDAPBC problem is solvable if there exist $H_{d}(x): \mathbb{R}^{n} \rightarrow \mathbb{R}$ satisfying (i), (ii) of Proposition 3.1 with $H_{a}(x)=H_{d}(x)-$ $H(x)$, matrices $J_{a}(x), R_{a}(x)$ and a feedback, $\bar{u}=\bar{u}(x)$ : $\mathbb{R}^{n} \rightarrow \mathbb{R}$ verifying the matching equation

$$
\begin{aligned}
& \left(\left(J_{d}(x)-R_{d}(x)\right)\right)\left(\left.\bar{\nabla} H\right|_{x} ^{x^{+}(\bar{u})}-\left.\bar{\nabla} H\right|_{x} ^{x^{+}}+\left.\bar{\nabla} H_{a}\right|_{x} ^{x^{+}(\bar{u})}\right) \\
& =g(x, \bar{u}) \bar{u}-\left.\left(J_{a}(x)-R_{a}(x)\right) \bar{\nabla} H\right|_{x} ^{x^{+}}
\end{aligned}
$$

with $J_{d}(x):=J(x)+J_{a}(x)=-J_{d}^{\top}(x), R_{d}(x):=R(x)+$ $R_{a}(x)=R_{d}^{\top}(x) \succeq 0$. Moreover, $x_{\star}$ is a stable equilibrium of the closed-loop system (2) with new Lyapunov function $H_{d}(x)$; it is asymptotically stable if the largest invariant set contained in $\left\{x \in \mathbb{R}^{n}||\left|\bar{\nabla} H_{d}\right|_{x}^{x^{+}(\bar{u})} \|_{R_{d}}^{2}=0\right\}$ is $\left\{x_{\star}\right\}$.

Proof. The matching equation (7) is deduced by equating (2) with the right-hand side of (1a) under feedback $u=\bar{u}(x)$ provided that $x_{\star} \in \mathcal{E}$. The control $u=\bar{u}+v$ makes (1a) a portHamiltonian dynamics of the form (2). The stability of $x_{\star} \in \mathcal{E}$ is ensured by (i), (ii) in Proposition 3.1 and the property 2) of the discrete Hamiltonian form as in Section II.A.

Remark 3.1: A necessary condition for the solvability of (7) is that there exists $\bar{u}(x)$ satisfying the matching condition

$$
\begin{aligned}
g^{\perp}(x, \bar{u})\left[\left.M_{a}(x) \bar{\nabla} H\right|_{x} ^{x^{+}}+M_{d}(x)\left(\left.\bar{\nabla} H\right|_{x} ^{x^{+}(\bar{u})}\right.\right. \\
\left.\left.-\left.\bar{\nabla} H\right|_{x} ^{x^{+}}+\left.\bar{\nabla} H_{a}\right|_{x} ^{x^{+}(\bar{u})}\right)\right]=0
\end{aligned}
$$

for all $x \in \mathbb{R}^{n}$ with $M_{a}(x)=\left(J_{a}(x)-R_{a}(x)\right), M_{d}(x)=$ $\left(J_{d}(x)-R_{d}(x)\right)$.

Remark 3.2: Albeit Proposition 3.1 characterizes all the possible solutions, the computability of $\bar{u}(x)$ remains challenging. Writing the discrete gradient in $O\left(\left\|x^{+}(u)-x^{+}\right\|^{2}\right)$

$$
\begin{aligned}
\left.\bar{\nabla} H\right|_{x} ^{x^{+}(u)}= & \left.\bar{\nabla} H\right|_{x} ^{x^{+}}+\frac{1}{2} \nabla^{2} H(x)\left(x^{+}(u)-x^{+}\right) \\
& +O\left(\left\|x^{+}(u)-x^{+}\right\|^{2}\right)
\end{aligned}
$$

with $x^{+}(u)-x^{+}=g(x, u) u$ and $g(x, u)=g(x, 0)+O(|u|)$, an approximate solution can be computed rewriting (7) as

$$
\left.M_{d}(x) \bar{\nabla} H_{a}\right|_{x} ^{x^{+}}+\left.M_{a}(x) \bar{\nabla} H\right|_{x} ^{x^{+}}=K_{d}(x) \bar{u}+O\left(|\bar{u}|^{2}\right)
$$

with $K_{d}(x)=\left(I_{d}-\frac{1}{2} M_{d}(x) \nabla^{2} H_{d}(x)\right) g(x, 0)$ so getting

$$
\bar{u}=K_{d}^{\dagger}(x)\left(\left.M_{a}(x) \bar{\nabla} H\right|_{x} ^{x^{+}}+\left.M_{d}(x) \bar{\nabla} H_{a}\right|_{x} ^{x^{+}}\right)
$$

and $\nabla^{2} H_{d}\left(x_{\star}\right) \neq 0$. 
Remark 3.3: The approximate solution highlighted in Remark 3.2 only yields local stability of the closed-loop equilibrium $x_{\star} \in \mathcal{E}$. However, global validity can be recovered by setting $\bar{u}_{a}(x)=\lambda(x) \bar{u}(x)$ with $\lambda(\cdot)>0$ suitably defined as in [22, Theorem 2.2].

\section{LineAR PORT-HAMILTONIAN SYSTEMS}

In this section, discussing the LTI case, necessary and sufficient conditions for solving the IDA-PBC problem are provided with a constructive solution for the control.

Assuming a quadratic Hamiltonian $H(x)=\frac{1}{2} x^{\top} P x$ with $P=P^{\top} \succ 0$ and $c \in \mathbb{R}$, the discrete gradient takes the form

$$
\left.\bar{\nabla} H\right|_{x} ^{x^{+}}=\frac{1}{2} P\left(x^{+}+x\right) .
$$

A LTI discrete port-Hamiltonian dynamics is described by

$$
x^{+}(u)=x+\frac{1}{2}(J-R) P\left(x^{+}+x\right)+B u
$$

with matrices $J=-J^{\top}, R=R^{\top} \succeq 0$ and $P=P^{\top} \succ 0$. The explicit state-space representation is computed by solving (9) in $x^{+}(0)=x^{+}$with $u=0$ so getting

$$
x^{+}(u)=A x+B u
$$

where $x^{+}(u)=x(k+1), x^{+}=A x$ and

$$
A=\left(I-\frac{1}{2}(J-R) P\right)^{-1}\left(I+\frac{1}{2}(J-R) P\right)
$$

with, by definition, $I-\frac{1}{2}(J-R) P$ nonsingular [20]. In this context, the set of admissible equilibria in Definition 2.3 for (9) specifies as $\mathcal{E}:=\operatorname{ker}\left\{B^{\perp}(J-R) P\right\}$.

Under this premise, the DT-IDA-PBC problem reduces in finding $u=\bar{u}+v$ which makes $x_{\star} \in \mathcal{E}$ stable for the closed-loop port-Hamiltonian system associated with the new Hamiltonian $H_{d}(x)=\frac{1}{2}\left(x-x_{\star}\right)^{\top} P_{d}\left(x-x_{\star}\right)$; namely,

$$
x^{+}(\bar{u}+v)=x+\frac{1}{2}\left(J_{d}-R_{d}\right) P_{d}\left(x^{+}(\bar{u})+x-2 x_{\star}\right)+B v
$$

with $J_{d}=J_{d}^{\top}, R_{d}=R_{d}^{\top} \succeq 0$, and $P_{d}=P_{d}^{\top} \succ 0$ and, equivalently in closed form,

$$
x^{+}(\bar{u}+v)=A_{d} x+A_{d, \star} x_{\star}+B v
$$

with $x^{+}(\bar{u})=A_{d} x+A_{d, \star} x_{\star}$ and

$$
\begin{aligned}
A_{d} & =\left(I-\frac{1}{2}\left(J_{d}-R_{d}\right) P_{d}\right)^{-1}\left(I+\frac{1}{2}\left(J_{d}-R_{d}\right) P_{d}\right) \\
A_{d, \star} & =-\left(I-\frac{1}{2}\left(J_{d}-R_{d}\right) P_{d}\right)^{-1}\left(J_{d}-R_{d}\right) P_{d} .
\end{aligned}
$$

The following result is deduced from Proposition 3.2 and provides a necessary and sufficient condition for solving the DT-IDA-PBC problem with matching equation (7) given by

$$
(J-R) \frac{P}{2}\left(x^{+}+x\right)+B \bar{u}=\left(J_{d}-R_{d}\right) \frac{P_{d}}{2}\left(x^{+}(\bar{u})+x-2 x_{\star}\right)
$$

or, equivalently, as

$$
A x+B \bar{u}=A_{d} x+A_{d, \star} x_{\star} .
$$

Theorem 4.1: Given the LTI dynamics (9), and $x_{\star} \in \mathcal{E}$, the DT-IDA-PBC problem is solvable for (9) if and only if there exist $J_{a}, R_{a}$ and $P_{a}$ solutions to

$$
B^{\perp}\left(J_{a}-R_{a}\right) P+B^{\perp}\left(J_{a}+J-R_{a}-R\right) P_{a}=0
$$

such that $J_{d}=\left(J+J_{a}\right)=-J_{d}^{\top}, R_{d}=\left(R+R_{a}\right)=R_{d}^{\top} \succeq 0$, and $P_{d}=\left(P+P_{a}\right) \succ 0$. In addition, the feedback assigning the port-Hamiltonian dynamics (11) (or, equivalently, (12)) with $x_{\star} \in \mathcal{E}$ is given by

$$
\bar{u}=B^{\dagger}\left\{M_{d} \frac{P_{d}}{2}\left(x^{+}(\bar{u})+x-2 x_{\star}\right)-M \frac{P}{2}\left(x^{+}+x\right)\right\}
$$

and in the explicit form, by

$$
\bar{u}=B^{\dagger}\left(A_{d}-A\right) x+B^{\dagger} A_{d, \star} x_{\star}
$$

with $A_{d}$ and $A_{d, \star}$ as in (13).

Proof. One must prove that (16) is necessary and sufficient for solving the matching equation (14). For necessity, by virtue of Remark 3.1, if the problem is solvable (i.e., (14) holds), then the matching condition (8), specified as

$$
B^{\perp}\left[M_{d} \frac{P_{d}}{2}\left(x^{+}(\bar{u})+x-2 x_{\star}\right)-M \frac{P}{2}\left(x^{+}+x\right)\right]=0,
$$

holds. By manipulating the equality above on gets

$$
B^{\perp}\left[M_{d} \frac{P_{d}}{2}-M \frac{P}{2}\right]\left(x^{+}+x\right)=-B^{\perp} M_{d} \frac{P_{d}}{2}\left(x^{+}(\bar{u})-x^{+}-2 x_{\star}\right)
$$

whereby the right-hand-side vanishes, because

$$
\begin{aligned}
& B^{\perp} M_{d} \frac{P_{d}}{2}\left(x^{+}(\bar{u})-x^{+}-2 x_{\star}\right)=B^{\perp}\left[\left(x^{+}(\bar{u})-x\right)-\left(x^{+}-x\right)\right] \\
& =B^{\perp}\left[x^{+}(\bar{u})-x^{+}\right]=B^{\perp} B \bar{u}=0,
\end{aligned}
$$

so getting $B^{\perp}\left[M_{d} P_{d}-M P\right]\left(x^{+}+x\right)=0$. By equivalence between (9) and (10), and rewriting $x^{+}+x=\left(A+I_{d}\right) x=$ $\left(I_{d}-\frac{1}{2} M P\right)^{-1} x$, one gets

$$
\begin{aligned}
& B^{\perp}\left[M_{d} P_{d}-M P\right]\left(x^{+}+x\right) \\
& =B^{\perp}\left[M_{d} P_{d}-M P\right]\left(I_{d}-\frac{1}{2} M P\right)^{-1} x=0
\end{aligned}
$$

which must hold for all $x \in \mathbb{R}^{n}$. By nonsingularity of $\left(I_{d}-\right.$ $\left.\frac{M P}{2}\right)^{-1}$, the above equality implies $B^{\perp}\left[M_{d} P_{d}-M P\right]=0$ that is (16) by definition of $M_{d}=J+J_{a}-R-R_{a}$ and $P_{d}=P+$ $P_{a}$. Then, necessity is proved. The sufficiency works out by showing that, if (16) holds, the feedback (17) (or, equivalently, (18)) solves the problem. To this end, plugging the feedback (17) into (14) one has

$$
\begin{aligned}
& B B^{\dagger}\left\{M_{d} \frac{P_{d}}{2}\left(x^{+}(\bar{u})+x-2 x_{\star}\right)-M \frac{P}{2}\left(x^{+}+x\right)\right\} \\
& =M_{d} \frac{P_{d}}{2}\left(x^{+}(\bar{u})+x-2 x_{\star}\right)-M \frac{P}{2}\left(x^{+}+x\right) .
\end{aligned}
$$

Since $B B^{\dagger}=I_{d}-\left(B^{\perp}\right)^{\top}\left[\left(B^{\perp}\left(B^{\perp}\right)^{\top}\right]^{-1} B^{\perp}\right.$, the equality above reduces to

$$
\begin{gathered}
-\left(B^{\perp}\right)^{\top}\left[( B ^ { \perp } ( B ^ { \perp } ) ^ { \top } ] ^ { - 1 } B ^ { \perp } \left\{M_{d} \frac{P_{d}}{2}\left(x^{+}(\bar{u})+x-2 x_{\star}\right)\right.\right. \\
\left.-M \frac{P}{2}\left(x^{+}+x\right)\right\}=0
\end{gathered}
$$

which holds true as $\left(J_{a}, R_{a}, P_{a}\right)$ satisfy (16) and (19). As a consequence, the feedback solution to (17) assigns the target 
dynamics (11) to (9). As far as the explicit form is concerned, plugging $u=\bar{u}+v$ into (10), with $\bar{u}$ as in (18), one gets (12).

We note that the matching condition (14) takes the form of a matrix equality.

Remark 4.1: The design can be addressed in two steps: first, one solves the linear equality (16) specifying the matching equation in the port-Hamiltonian formalism; then, the feedback of the form $\bar{u}=F x+F_{\star} x_{\star}$. with $F=B^{\dagger}\left(A_{d}-A\right)$ and $F_{\star}=B^{\dagger} A_{d, \star}$ can be exactly computed making reference to the explicit representations associated to (9) and (11).

\section{IDA-PBC FOR SAMPLED LTI MECHANICAL SYSTEMS}

The proposed discrete IDA-PBC strategy is applied to sampled-data stabilization of a linear mechanical system.

As discussed in [4], LTI mechanical systems involve quadratic potential energy and constant inertia so that the Hamiltonian is given by $H(q, p)=\frac{1}{2} p^{\top} M^{-1} p+\frac{1}{2} q^{\top} K q$ with mass $M \succ 0$, stiffness $K \succ 0$ and momentum $p=M \dot{q}$, with $(q, p)$ denoting the generalized coordinates. For the sake of simplicity assume that the continuous-time LTI mechanical system evolves in $\mathbb{R}^{2}$. The associated port-Hamiltonian dynamics is given by

$$
\dot{x}=\left(\begin{array}{cc}
0 & 1 \\
-1 & 0
\end{array}\right) \nabla H(x)+\left(\begin{array}{c}
0 \\
G
\end{array}\right) u
$$

with $x=\operatorname{col}(q, p) \in \mathbb{R}^{2}$ and $G \in \mathbb{R}$. The origin of (20) is stable and the admissible equilibrium $x_{\star}$ is contained in $\mathcal{E}:=\operatorname{span}\{\operatorname{col}(\bar{q}, 0)\}, \forall \bar{q} \in \mathbb{R}$.

\section{A. Sampled-data equivalent representation}

The equivalent sampled-data model of (20) can be computed exploiting the exact discretization proposed in [20].

Proposition 5.1: Consider the continuous-time system (20), then there exists $T^{\star}>0$ (the maximum allowable sampling period) such that for any $\delta \in] 0, T^{\star}[$ its sampled equivalent port-Hamiltonian dynamics (9) is

$$
x^{+}(u)=x+\frac{1}{2} J^{\delta} P\left(x^{+}+x\right)+B^{\delta} u
$$

with $x^{+}=x^{+}(0)=A^{\delta} x, \omega=\sqrt{\frac{K}{M}}$

$$
\begin{aligned}
& J^{\delta}=\left(\begin{array}{cc}
0 & \frac{\sin (\omega \delta)}{\omega(1+\cos (\omega \delta))} \\
-\frac{\sin (\omega \delta)}{\omega(1+\cos (\omega \delta))} & 0
\end{array}\right), P=\left(\begin{array}{cc}
K & 0 \\
0 & M^{-1}
\end{array}\right) \\
& B^{\delta}=\left(\begin{array}{cc}
\frac{1}{K}(1-\cos (\omega \delta)) \\
\frac{1}{\omega} \sin (\omega \delta)
\end{array}\right), A^{\delta}=\left(\begin{array}{cc}
\cos (\omega \delta) & \frac{\omega}{K} \sin (\omega \delta) \\
-M \omega \sin (\omega \delta) & \cos (\omega \delta)
\end{array}\right) .
\end{aligned}
$$

Proof. The proof follows the line of [20, Theorem 4.18]. Rewriting (20) as $\dot{x}=J P x+B u$, then $B^{\delta}=\int_{0}^{\delta} e^{\tau J P} \mathrm{~d} \tau B$, $A^{\delta}=\left(I-\frac{1}{2}\left(J^{\delta}-R^{\delta}\right) P\right)^{-1}\left(I+\frac{1}{2}\left(J^{\delta}-R^{\delta}\right) P_{d}\right)$, and $J^{\delta}=$ $\frac{2}{\delta}\left(e^{\delta J P}-I_{d}\right)\left(e^{\delta J P}+I_{d}\right)^{-1} P^{-1}$.

The discrete-time model (21), parametrized by $\delta$, represents the exact sampled-data equivalent model of (20). In particular, it possesses the discrete port-Hamiltonian structure (9) and, contrarily to standard models adopted in the literature, preserves, for all $t=k \delta, k \geq 0$ and $\delta \geq 0$, the same energetic properties as the continuous-time one in (20). For these reasons, (21) is instrumental to compute a piecewise constant control assigning $x_{\star} \in \mathcal{E}$ to (20) via DT-IDA-PBC.

\section{B. Discrete IDA-PBC design}

Applying Theorem 4.1, the mechanical system can be stabilized at $x_{\star}=\operatorname{col}(\bar{q}, 0)$ through discrete-time IDA-PBC once selected $J_{a}, R_{a}, P_{a}$ so that (16) specifies as

$$
B^{\delta \perp}\left[\left(J_{a}^{\delta}-R_{a}^{\delta}\right) P-\left(J^{\delta}+J_{a}^{\delta}-R^{\delta}-R_{a}^{\delta}\right) P_{a}\right]=0,
$$

with $B^{\delta \perp}=\alpha\left(\frac{1}{\omega} \sin (\omega \delta) \quad K \cos (\omega \delta)-K\right)$ for any $\delta \in$ ] $0, T^{\star}\left[\right.$ and $\alpha \in \mathbb{R}$. Fixing $J_{a}^{\delta}=0$, the admissible equilibrium $x_{\star}=\operatorname{col}(\bar{q}, 0)$ can be assigned to (20) under the choice

$$
\begin{aligned}
P_{a} & =\left(\begin{array}{cc}
0 & 0 \\
0 & P_{s}
\end{array}\right), \quad \text { with } P_{s}=\frac{1}{M} \tilde{S} \\
R_{a}^{\delta} & =\left(\begin{array}{cc}
\omega(1-\cos (\omega \delta)) & \frac{1}{K} \sin (\omega \delta) \\
\frac{1}{K} \sin (\omega \delta) & \frac{1}{K \omega}\left(1+\cos (\omega \delta)-\frac{1}{\omega} \frac{\tilde{S}}{\tilde{S}+1}\right)
\end{array}\right)
\end{aligned}
$$

where $\tilde{S}$ is freely chosen so that $-1 \leq \tilde{S} \leq \frac{\rho}{1-\rho}$ with $\rho=$ $\omega \frac{K-1}{K}(1+\cos (\delta))$ so to guarantee $P_{d} \succ 0$. Finally, the IDAPBC control solution to (17) is given by

$$
\bar{u}=B^{\delta \dagger}\left[\left(A_{d}^{\delta}-A^{\delta}\right) x+A_{d, \star}^{\delta} x_{\star}\right]
$$

with assigned dynamical matrices

$$
\begin{aligned}
& A_{d}^{\delta}=\left(I-\frac{1}{2}\left(J^{\delta}-R_{a}^{\delta}\right) P_{d}\right)^{-1}\left(I+\frac{1}{2}\left(J^{\delta}-R_{a}^{\delta}\right) P_{d}\right), \\
& A_{d, \star}^{\delta}=-\left(I-\frac{1}{2}\left(J^{\delta}-R_{a}^{\delta}\right) P_{d}\right)^{-1}\left(J^{\delta}-R_{a}^{\delta}\right) P_{d} .
\end{aligned}
$$

Remark 5.1: The proposed solution is based on a direct discrete-digital design and differs from the continuous-time one as it requires constructive matrices $R_{a}^{\delta}$ and $P_{a}^{\delta}$ induced by the annihilator $B^{\delta \perp}$. This ad hoc direct-digital design also differs from those proposed in [9]-[11], [17] which strictly rely upon matching of the continuous-time dissipation and an approximate Hamiltonian form of the discrete-time model.

\section{Simulation results}

Simulations show the asymptotic stabilization of $x_{\star}=$ $\operatorname{col}(2,0)$ of the system (20). Setting $M=K=1$ and $x_{0}=\operatorname{col}(20,10)$, Figure 1 and Figure 2 depict the closed-loop behavior and the control effort of the proposed IDA-PBC in (22) (blue), the standard discrete IDA-PBC in [10] (red), and the continuous-time IDA-PBC in [4] (black) and its emulationbased controller (green).

Figure 1a shows that as far as $\delta$ is small enough asymptotic stabilization of $x_{\star}$ is achieved under any one of the controllers; in particular the emulated (green) and the standard discrete controllers (red) provide trajectories which are close to the continuous-time ones (black), while the proposed controller (blue) provides a different behavior (as it is a direct digital design). As $\delta$ increases, the emulated control fails (Figure 1b$2 \mathrm{~b}$ ); moreover at $\delta=2.3375$, the literature control makes the corresponding trajectories fall into a limit cycle (Figure 1c) until failing in stabilizing as $\delta$ increases (Figure 1d-2d); such a cycle is due to the assignment to the discrete model of (20) 


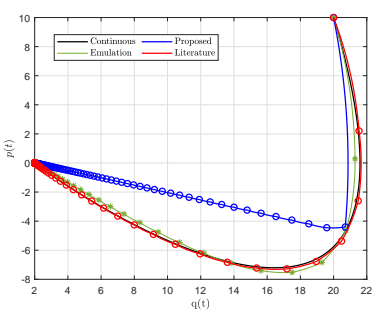

(a) $\delta=0.25$

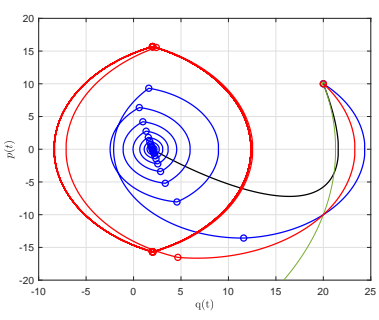

(c) $\delta=2.3375$

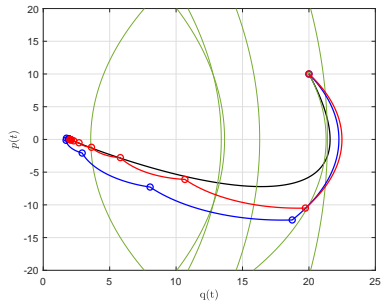

(b) $\delta=1$

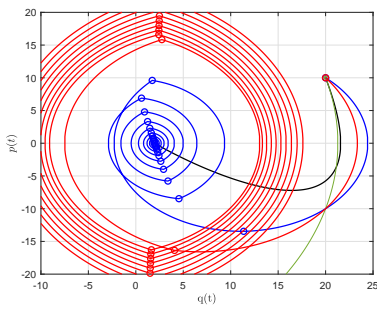

(d) $\delta=2.38$
Fig. 1. Phase-portrait of (20) with IDA-PBC (22) (blue), discrete IDA-PBC in [10] (red), continuous IDA-PBC in [4] (black) and its emulated (green).

of one eigenvalue in $\lambda_{1}=-1$ and the other with $\left|\lambda_{2}\right| \leq 1$. The effectiveness of the proposed controller is clear as it still stabilizes $x_{\star}$.

\section{CONCLUSION}

A novel IDA-PBC strategy for discrete port-Hamiltonian dynamics is proposed. Although a solution to the implicit matching equation is challenging to determine, a novel approach is proposed in the nonlinear context and fully developed in the LTI case. It is shown through an example that the proposed strategy asymptotically stabilizes the admissible equilibrium for any sampling period $\delta$. Perspectives concern sufficient conditions for satisfying the matching equality based on approximations of the discrete gradient.

\section{REFERENCES}

[1] B. M. Maschke and A. J. van der Schaft, "Port-controlled Hamiltonian systems: modelling origins and system theoretic properties," IFAC Proceedings Volumes, vol. 25, no. 13, pp. 359-365, 1992.

[2] A. van der Schaft and D. Jeltsema, "Port-Hamiltonian systems theory: An introductory overview," Foundations and Trends® in Systems and Control, vol. 1, no. 2-3, pp. 173-378, 2014.

[3] R. Ortega, A. Van Der Schaft, B. Maschke, and G. Escobar, "Interconnection and damping assignment passivity-based control of portcontrolled Hamiltonian systems," Automatica, vol. 38, no. 4, pp. 585596, 2002.

[4] R. Ortega, Z. Liu, and H. Su, "Control via interconnection and damping assignment of linear time-invariant systems: a tutorial," International Journal of Control, vol. 85, no. 5, pp. 603-611, 2012.

[5] B. Brogliato, R. Lozano, B. Maschke, and O. Egeland, "Dissipative systems analysis and control," Theory and Applications, vol. 2, 2007.

[6] S. Stramigioli, C. Secchi, A. J. van der Schaft, and C. Fantuzzi, "Sampled data systems passivity and discrete port-Hamiltonian systems," IEEE Transactions on Robotics, vol. 21, no. 4, pp. 574-587, 2005.

[7] D. S. Laila and A. Astolfi, "Discrete-time IDA-PBC design for separable Hamiltonian systems," IFAC Proceedings Volumes, vol. 38, no. 1, pp. 838-843, 2005.

[8] — , "Discrete-time IDA-PBC design for underactuated Hamiltonian control systems," in American Control Conference. IEEE, 2006, pp. $188-193$.

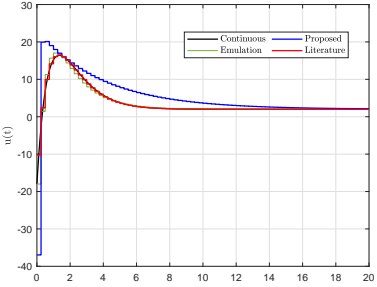

(a) $\delta=0.25$

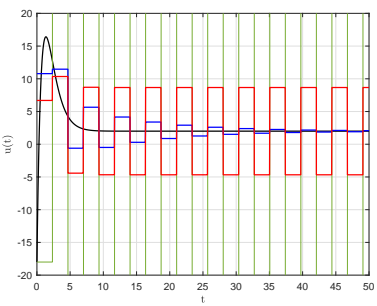

(c) $\delta=2.3375$

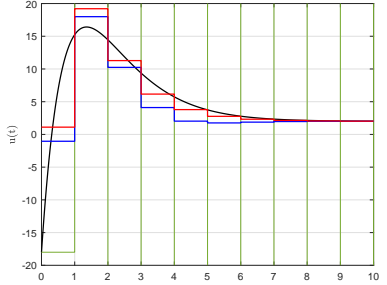

(b) $\delta=1$

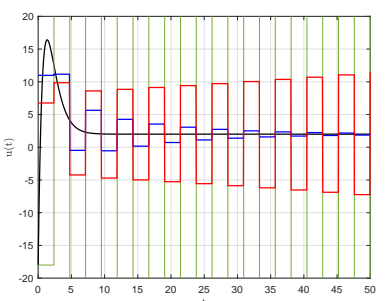

(d) $\delta=2.38$
Fig. 2. Time histories of the IDA-PBC (22) (blue), discrete IDA-PBC in [10] (red), continuous IDA-PBC in [4] (black) and its emulated (green).

[9] L. G. Sümer and Y. Yalçin, "A direct discrete-time IDA-PBC design method for a class of underactuated Hamiltonian systems," IFAC Proceedings Volumes, vol. 44, no. 1, pp. 13 456-13 461, 2011.

[10] S. Aoues, D. Eberard, and W. Marquis-Favre, "Discrete ida-pbc design for $2 \mathrm{~d}$ port-hamiltonian systems," IFAC Proceedings Volumes, vol. 46 , no. 23, pp. $134-139,2013$.

[11] _ "Discrete IDA-PBC control law for Newtonian mechanical portHamiltonian systems," in 54th IEEE Conference on Decision and Control (CDC), 2015, pp. 4388-4393.

[12] V. Talasila, J. Clemente-Gallardo, and A. van der Schaft, "Discrete portHamiltonian systems," Systems \& Control Letters, vol. 55, no. 6, pp. 478-486, 2006.

[13] Y. Yalçin, L. G. Sümer, and S. Kurtulan, "Discrete-time modeling of Hamiltonian systems," Turkish Journal of Electrical Engineering \& Computer Sciences, vol. 23, no. 1, pp. 149-170, 2015.

[14] S. Aoues, M. Di Loreto, D. Eberard, and W. Marquis-Favre, "Hamiltonian systems discrete-time approximation: Losslessness, passivity and composability," Systems \& Control Letters, vol. 110, pp. 9-14, 2017.

[15] T. Itoh and K. Abe, "Hamiltonian-conserving discrete canonical equations based on variational difference quotients," Journal of Computational Physics, vol. 76, no. 1, pp. 85-102, 1988.

[16] M. De Stefano, R. Balachandran, A. M. Giordano, C. Ott, and C. Secchi, "An energy-based approach for the multi-rate control of a manipulator on an actuated base," in IEEE International Conference on Robotics and Automation, May 2018, pp. 1072-1077.

[17] S. Monaco, D. Normand-Cyrot, and F. Tiefensee, "Nonlinear port controlled Hamiltonian systems under sampling," in 48h IEEE Conference on Decision and Control (CDC) held jointly with 28th Chinese Control Conference. IEEE, 2009, pp. 1782-1787.

[18] A. Moreschini, M. Mattioni, S. Monaco, and D. Normand-Cyrot, "Discrete port-controlled hamiltonian dynamics and average passivation," in 58th IEEE Conference on Decision and Control (CDC), 2019, pp. 14301435.

[19] S. Monaco and D. Normand-Cyrot, "Nonlinear average passivity and stabilizing controllers in discrete time," Systems \& Control Letters, vol. 60 , no. 6 , pp. 431-439, 2011

[20] A. Moreschini, S. Monaco, and D. Normand-Cyrot, "Gradient and Hamiltonian dynamics under sampling," IFAC-PapersOnLine, vol. 52, no. 16, pp. 472-477, 2019.

[21] R. I. McLachlan, G. Quispel, and N. Robidoux, "Geometric integration using discrete gradients," Philosophical Transactions of the Royal Society of London. Series A: Mathematical, Physical and Engineering Sciences, vol. 357, no. 1754, pp. 1021-1045, 1999.

[22] M. Mattioni, S. Monaco, and D. Normand-Cyrot, "Forwarding stabilization in discrete time," Automatica, vol. 109, pp. 108532, 2019. 